water. To-day somewhat distended. Has passed a great deal of gas by rectal tube, unable to pass wind unaided. As the gas accumulates causes much discomfort, which is immediately relieved by its passage. Milk and lime-water nauseates, hence oatmeal gruel.

For a number of days difficulty with gas, requiring rectal tube and enemata. Soft solids on fourth, and house diet on sixth day after operation. Except for slight infection of stitches convalescence uninterrupted, and patient discharged on the sixteenth day after operation, with abdominal support, bowels moving daily with a mild laxative; no pain.

\section{CLEFT PALATE.}

Dr. C. B. Porter also presented a number of operations for cleft palate.

Case 1. This young man had cleft palate and had also been operated upon for hare-lip, the result of which certainly was not as good as that following my operation. The cleft in the palate is closed completely and he has had a bridge made in front to fill up the hiatus of the teeth gone so that it makes a most presentable lip and mouth. He is twenty. I did the first operation eighteen months ago.

CASE II. This young man is now undergoing some elocutionary gymnastics and his speech will undoubted!y improve a good deal though it is now much better than before operation. $\mathrm{He}$ has only been operated a few months, and the day after he was operated he developed diphtheria together with my assistant who was present at the operation, and that delayed his recovery, although the palate has united entirely. He is twenty-two.

CASE III. I operated on this patient five years ago. 'This case is really very successful indeed. I think if he had the small hiatus in front filled with teeth like Case $I$ he would get rid of the slight whistle heard at times as he can carry his palate close back against the posterior wall of the pharnyx. It seems to me when he gets this little fissure closed he will speak perfectly plain.

I would like to say a few words in regard to the operation, and that is that the angle made by the two halves of the hard palate is usually very acute. Instead of having an arch the two sides come in at an angle and when the soft parts are dissected off from the hard palate it is difficult to pass the sutures in the anterior part of the fissure and in the last two operations $I$ have cut the soft parts free in front so that the two flaps hang down into the mouth, then the sutures are placed with ease. I use silk sutures soaked in compound tincture of benzoin, which prevents them from slipping when tied. I also insist on an obturator like a plate for artificial teeth, to protect the wound from pressure of the tongue in swallowing, that meaus that I never operate unless there are teeth enough to which to fasten the obturator. I operate under ether and in the Rose position.

atonic dilatation OF STOMACH: GASTRORRHAPHY.

Dr. F. C. Shattuck: The patient, a Gloucester fisherman, forty-seven years old, entered the Massachusetts General Hospital, January 18, 1897.

Brother died of heart disease.

Had used no alcohol or tobacco. Denied venereal disease. Had usual children's diseases, measles at twenty-four, "slow fever" at nineteen, for three months, no cough.
The present illness began in 1883. Had, about once a year, periods of vomiting, with nausea and inability to retain food on stomach. These lasted for a week to two months, and kept him from work. Attack preceded several days by gastric distress and water-brash. Would usually lose twenty to fifty pounds, which was quickly regained after stomach symptoms subsided. Each attack was worse than the one preceding, both in severity and duration.

For the last five years the attacks have come twice a year and usually last a mouth. A year ago he was ill three months, and lost forty pounds in weight, which he regained in the next two months. After this his health was very good until last May, when the old trouble again returned. Since then he has vomited daily, and pain and distress in stomach have increased.

Ten weeks ago, while out in a fishing schooner, the vomitus for a fortnight was like coffee grounds. From that time he has done practically no work, but has not been confined to bed. Recently, as a rule, he is comfortable during the day, but toward evening is taken with a gnawing pain in the epigastrium which gradually increases until he vomits in the middle of the night. The pain gradually wears awav.

Vomitus varies in quantity from a pint to a gallon, yellow or dark colored, very sour smelling, often containing undigested food.

Patient has marked constipation : he may have no movement for a week. 'The dejections are occasionally black and tarry, usually not.

There are eructations of gas and acids. Borborygmus. Appetite is fairly good.

His best weight was 200 pounds; he now weighs 148 pounds.

Physical Examination. Large skeleton; cheek-bones prominent. Tongue pale, Habby, heavily coated. Pulse strong, voluminous and forcible. Lungs vegative. Heart: small area of dulness; no murmurs. Liver: fifth rib, no edge felt. Inguinal glands of good size. Epitrocblear glands enlarged. Knee-jerks active. Urine: normal color, alkaline, $1.018 \frac{1}{2}$, no sugar, no albumin, no diazo, sediment heavy, amorphous phosphates. Blood: reds, 3,536,000; whites, 9,400 ; hemoglobin, 48 per cent.

Diet : Liquids and soft solids.

January $19 \mathrm{tb}$. This evening vomited 37 ounces of yellow fluid. Vomitus contained hydrochloric acid. Digestion leucocytosis present. At 1 P. M., proteid meal : whites, 9,550 ; at 4.30 P. M., whites, 12,800 ; gain of 3,250 .

January 20th. House diet with care. Liquids limited to one pint daily. Stomach washed after test-meal. Capacity, 3,000 c. c. Hydrochloric and lactic acids present, the latter in small amount. When inflated, lower border nearly reaches to pubes. No tumor to be felt. Daily washing of stomach before breakfast showed average residue of 15 ounces.

February 3d. Owing to dilatation of stomach, presence of hydrochloric acid, absence of tumor, and variations in weight, a diagnosis was made of atonic dilatation of the stomach; and after consultation with Dr. Porter the operation of gastrorrhapy was advised, the patient to return if operation was decided upon.

Patient entered the Surgical Ward for operation on February 15, 1897. Examined again by Dr. Shattuck. Stomach washed out. Dilatation same as before.

February 18th. Stomach washed out in morning. 
Washings clear; slight greenish tinge; vomitus contained hydrochloric acid, no lactic acid. Transferred to Ward E, for

OPERATION BY DR. PORTER.

Incision along border of ribs on left side, which reached from middle line just below ensiform cartilage to angle of ribs. Peritoneal cavity opened. Hand was introduced, and stomach found immediately below incision. Pylorus palpated, and a thickened mass felt in connection with it. Stomach pulled up to the incision. Some adhesions about pylorus and some about cardiac end made this very difficult. The stomach wall very slippery - scarcely to be held ex-

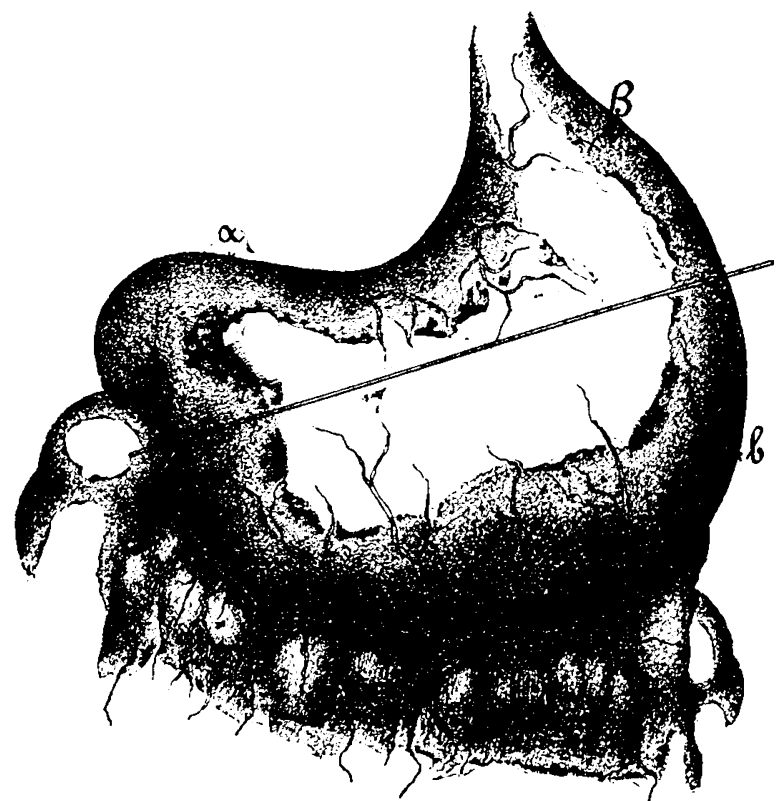

Fra. 1. - Dilated stomach, showing line of fold; $a$ to be sutured to $a$ and $b$ to $b$ with intermediate sutures.

cept with gauze - owing to the violent muscular contractions induced by the manipulation. When the stomach was at last delivered through the incision, more careful examination showed that the mass before felt was apparently behind the pylorus, and not a thickening or growth in its walls. Few enlarged glands found. One, size of large bean, removed from the omentum. Was examined immediately by Dr. Whitney and pronounced non-malignant, simply hyperplastic.

As there was some doubt about the mass near the pylorus, an incision was made through anterior wall of stomach, which was somewhat thickened. Index finger was introduced easily through pylorus, the mass lying behind and not connected with it. The incision in stomach was closed by two rows of catgut sutures through the mucous and muscular walls, a third row of Lembert silk sutures in the peritoneum; surface washed off with sterile water, and the gauzes which had been placed about this part of the stomach before the incision changed. The cardiac end of stomach was next pulled down as much as possible and one or two Lembert sutures placed in such a way that when drawn tight a part of the wall would be folded in. This was accomplished by making the space between the places where the suture passed into the wall of the stomach as wide as possible. A few similar sutures were then placed about the middle of the greater curvature. 'The pyloric end of the stomach was then pulled up as far as possible and the same sutures passed. 'These were to be used as guides, and were so placed that when tied they would bring the line of the greater curvature as high up towards the lesser curvature as possible - perhaps one and one-half to two inches below it. These guide sutures were then tied, and the fold thus made was beld invaginated while its edges were caught all the way along by a row of interrupted Lembert sutures. Holding the stomach during the last part of the operation was extremely difficult, as its muscular action seemed to have been excited by the manipulations. After the suturing, stomach allowed to retract into the abdominal cavity. No bleeding. Abdominal incision closed. Dressing, swathe. In good condition.

As soon as in bed, an enema of coffee and brandy,

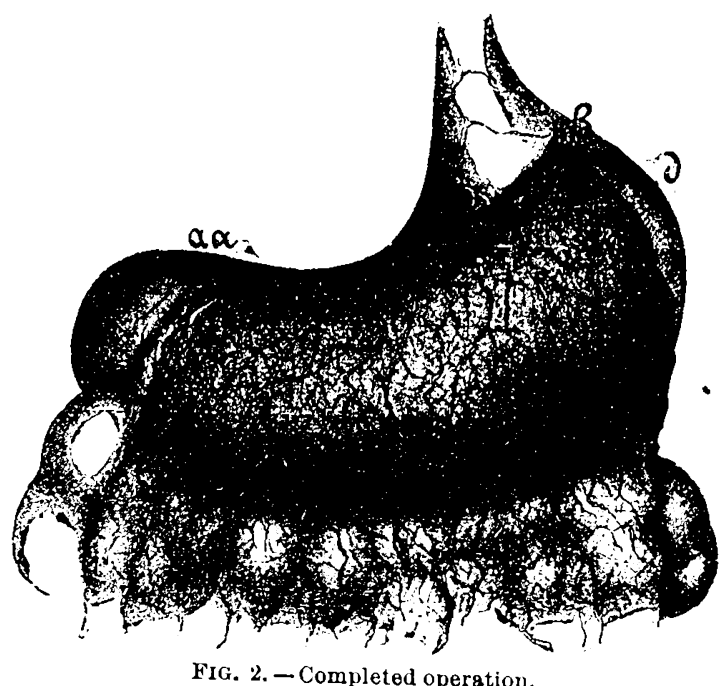

each two ounces. Salt one pint. During afternoon, a subcutaneous injection of strychnia (grain $\frac{1}{40}$ ). Patient to have absolutely nothing by mouth, nutritive enema every six hours. Beef-juice; peptonized milk, two ounces; egg albumin, one ounce; whiskey and salt solution, each one-half ounce. Soap and glycerine enema every forenoon to clean out rectum.

February 19th. Morphia (grain $\frac{1}{4}$ ) by subcutaneous injection last night. Patient fairly comfortable to-day. No vomiting; little pain.

February 20th. Second day. Complains of feeling nervous. Has had no vomiting since operation, not even during recovery from ether. Spits up a little to-day. Morphia (grain $\frac{1}{8}$ ) subcutaneously, and 30 grains of bromide in 9 P. r. enema. Seen by Dr. Porter in the evening. As patient seems to have lost ground a little, decided to start with mouth feeding. Half.drachm doses every one-half hour of albumin and water, beef-juice, whiskey, soda water, black coffee. Nutritive enemata every three hours.

February 21st. Took nourishment well during night. Nauseated but slightly on two occasions. This morning doses increased to one drachm every half hour. Enemata continued, with laudanum, five grains.

February $22 \mathrm{~d}$. No nausea. This morning, at 9 A. M., enema not retained, interval increased to six hours. 
February 23d. During the night feeding gradually perhaps forty-five years of age. In the anterior abincreased, so that this morning he is getting two ounces of liquids every hour.

February 24th. For a short time has been raising yellow and extremely foul-smelling sputum. Seen today by Dr. Shattuck, who finds trouble in bases of both lungs behind. Dulness and whispered bronchophony. One ounce of whiskey every three hours. Abdominal wound looks all right. Codeia (grain $\frac{1}{8}$ ) every four hours p. r. n. for cough.

February 26th. Nine days after operation. Says he feels much better to-day. Expectoration about the only thing of which be complains. Scrambled

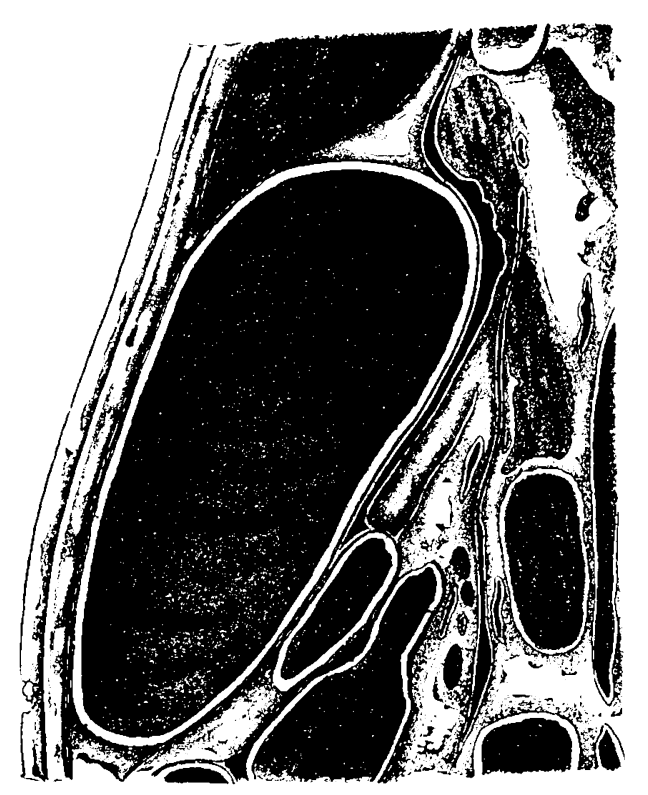

FrG. 3. - Vertical section of dilated stomach. dominal wall a recently healed wound, with suture marks, about $14 \mathrm{~cm}$. long, extending from a point $1 \frac{1}{2}$ $\mathrm{cm}$. below and from a point $1 \frac{1}{2} \mathrm{~cm}$. to right of ensiform cartilage toward the left, ruuning parallel to, and one or two centimetres below, costal margin.

No rigor. No scars or pains. Subcutaneous fat small in amount.

Abdominal wound apparently completely healed. Some adhesions of omentum in neighborhood. Peritoneal cavity free from fluid. Intestines, especially colon in its upper portions, widely distended with gas. Serosa of intestine smooth and shining. No injection.

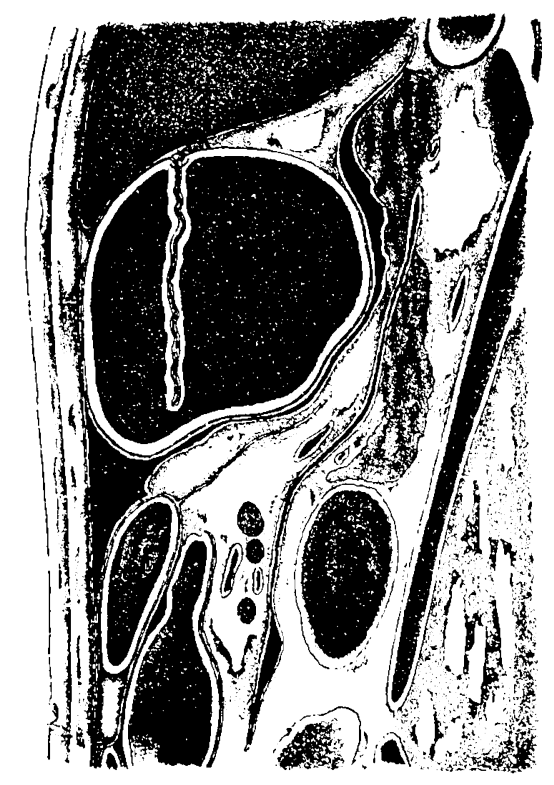

Fra. 4. - Vertical section of stomach after operation showing infolded wall.

Stomach adherent to liver and to diaphragm by a thin fibrinous exudate. The lesser peritoneal cavity not obliterated.

Liver adherent to diaphragm by fibrinous-like strings. In the neighborhood of gall-bladder old adhesions with colon.

In anterior wall of stomach there is a row of sutures along the long axis of the organ $13 \mathrm{~cm}$. long. Near pylorus a second row of sutures about $3 \mathrm{~cm}$. long. No evidence of suppuration. No escape of gas or fluid on pressure.

In both thoracic cavities a very small amount of fluid containing yellowish shreds.

Left Lung. Superior lobe extremely pale. On section, contains a moderate amount of a frothy, slightly reddish fluid. Inferior lobe largely collapsed. Its inferior portion, over an area corresponding perhaps to one-fourth of the lobe, near pleura, is broken down into dirty, grayish, stinking diffluent material, rather sharply marked out from lung tissue in neighborhood. The pleura of the inferior lobe and of the posterior inferior portion of the superior lobe covered with a fibrinous exudate which in places is yellow, several millimetres thick, elastic and infiltrated with fluid. The pleura in these situations firmly adberent to visceral pleura, on which a similar exudation is present, f most marked over the diaphragm, apparently opposite 
to the gangrenous area of the lower lobe. Just beneath the parietal pleura near the junction of the lower ribs with the spinal column there is a half-dollar-sized flattened elevation with circular outline covered with fibrinous exudate and on section composed of a redblack material resembling clotted blood. There was no perforation of diaphragm. The fibrinous exudate of pleura in places on the lung shows numerous minute vessels extending into it from the pleura.

Right Lung. In superior lobe a chestnut-sized area just beneath the pleura in which the lung tissue is broken down into a brownish-gray diffluent stinking material rather sharply marked out from surrounding lung tissue. In inferior lobe on pleura are two circular areas covered with a dirty, yellowish-gray exudate, rather sharply defined and varying in size from a halfdollar to a dollar. These circular areas seem to correspond to similar circular yellow elevations beneath parietal pleura, which on section are made up of a dirty gray diffluent purulent-looking necrotic material. They are situated in the posterior portion of the thoracic wall. A rib passing beneath one of them was excised and gave no evidence of suppuration in the intercostal or osseous tissue beneath. The areas in the pleura correspond to extensive areas of lung tissue transformed into a brownish-gray stinking diffluent gangrenous material. The areas are sharply marked off from surrounding lung tissue. The inferior lobe is markedly collapsed ; its pleura and the pleura of the posterior and inferior portions of the upper lobe covered with a fibrinous exudate which in places is yellow, thick and infiltrated with fluid. The tissue of the superior and middle lobes is extremely pale, not consolidated and contains a small amount of fluid. In lower lobe there is apparently some consolidatiou in the neighborhood of one of these gangrenous areas, the tissue being resistant, grayish and homogeneous. The pulmonary arteries leading to the inferior lobes of both lungs were dissected, but no good evidence of embolism could be made out. Bronchi injected, and contain a considerable amount of a dirty grayish opaque fluid.

Stomach. The long row of sutures corresponds to the infolding of the stomach wall, the short row to a wound in the stomach wall with inversion of the edges and coaptation of the sound edges. At the pylorus there is a distinct loss of substance in the mucous membrane in the form of an oval ulcer, five centimetres long by two centimetres wide and perbaps four or five millimetres deep. Its margins smooth, to a considerable extent undermined and the mucosa projecting over. The floor fairly smooth with three to four small polypoid projections. The edge of the ulcer reaches to within one or two millimetres of the pylorus. The tissue in the neighborhood of the ulcer not especially indurated nor is the stomach wall especially thickened abuut it. The pylorus admits the index finger. The stomach, as it appears with the pleat in its wall, is of moderately large dimensions. The stomach contents show no evidence of hemorrhage. The nucosa not remarkable. Other organs not remarkable.

Anatomical Diagnosis. Ulcus ventriculi, with dilatation of stomach; gastrorrhaphy; gastrotomy ; circunscribed fibrinous peritonitis; gangrene of lungs; fibriuous pleuritis.

Bacteriological report. Cultures in blood serum; \begin{tabular}{r|l} 
spleen, liver, kidneys, and left side of the diaphragm & or secondary lesions in the family. As is often the \\
case, the importance of the late manifestations are
\end{tabular} sterile; heart: one medium-sized, round, whitish, shining colony made up of staphylococcus pyogenes albus. Right pleura: Four colonies of staphylococcus pyogenes albus, and one of staphylococcus pyogenes aureus. Gangrene of lung: A fairly large number of colonies of staphylococcus pyogenes aureus; also numerous small, round, moist, colonies made up of streptococci.

Dr. ShatTdCK: It seems peculiarly hard that this patient above all others should have succumbed to deglutition pneumonia because his stomach was washed out the morning before operation.

Dr. Wright: The autopsy showed that the cause of death was gangrene of the lungs. The stomach itself presented the greatest interest. I have here the specimen, hardened in formaline. This is the anterior aspect of the stomach wall. Here is the esophagus; here is the pyloric end. Along here you see a row of sutures, and at this point another row of sutures near the pylorus. When we open the stomach we find that the long row of sutures corresponds to an invagination of the stomach wall along this line, and at the pyloric end of the stomach we see a round ulcer about the size of a quarter-dollar, with smooth margins, a rather indurated base, and no extensive thickening of the stomach wall; in other words, a simple ulcer of the stomach situated exactly on the margin of the pylorus. At the autopsy I could find no evidence of stricture of the organ, but it seemed to me very probable that this ulcer might have interfered very materially with the working of the pylorus. The bacteriological examination of the various organs showed infection which proceeded from the lungs.

\section{TWO CASES OF AORTIC REgURgitation AND TABES DORSALIS.}

DR. H. F. VickerY : I desire to show two patients who came to me in the Out-Patient Department. The first one who appeared was the father of the second plained of some stomach trouble. Incidentally, in examining him, it was noticed that there were enlarged glands in the neck and at the elbows, and that the pulse was of high tension, with the aortic second sound sharp and accentuated, without any cardiac murmur. He now presents the well-marked symptoms of an aortic regurgitation and of tabes dorsalis. With regard to the origin of the trouble, we have no history of any primary lesion. He acknowledges venereal exposure.

The little girl, who is now approaching fourteen children bave been still-born very near term. She was brought for a choreiform condition; she was nervous and fussy, and would drop things. She has improved a good deal. Other things to be noticed about her are as follows: around the angles of the mouth there is a scar-like condition; as her profile is seen, the bridge of the nose shows an abnormal depression, the frontal bone is large and the frontal eminences are well-marked; the upper central incisors are distinctly peg-shaped; there is evidence of a former keratitis.

All these things exist without a history of primary case, the importance of the late manifestations are one. Two years ago, at the age of thirty-six, he comyears, is perhaps rather small for her age. She was the result of the first pregnancy; and since then two 